\title{
Syzygium aromaticum : A subtropical tree scrutinized for the genetic differentiation among the altitudes of Nilgiris
}

Saranya D.* and R. Ravi

PG and Research Department of Botany, Government Arts College, Ooty, The Nilgiris, TamilNadu, India.

Received:3/8/2018; Accepted:3/20/2018

\begin{abstract}
Clove (Syyygium aromaticum) is one of the most economically significant oil producing trees in India, whose importance is mainly due to its medicinal features. Unfortunately, importing rate of the spice is increasing which would be an economic drawback for the country. Therefore, information regarding genetic diversity and the oil quality of the tree is necessary as a part to increase the cultivation. Thus the present work was conducted to identify the genetic difference between the collected samples among different altitudes of Nilgiris in comparison to the extracted oil quality to select high quality oil yielding variety for cropping. RAPD and GC-MS techniques were used for analyzing genetic diversity and oil extraction respectively. The phylogeny constructed revealed that the samples S1\& S6 are genetically more similar, whereas S2 is far distinct from all other samples. S3 shared a close relation with S1 \& S6. Samples S4, S5 were separated out but are within the same node. The eugenol percentage of the samples S1, S2, S3, S4, S5 and S6 are $42.96 \%, 68.78 \%, 55.95 \%, 71.17 \%, 74.04 \%$ and $73.47 \%$ respectively. From the collected data it is inferred that though the samples shared genetic similarity there observed a considerable difference in the eugenol content. This information needs to be correlated with the cultivation practices and the soil nutrition based on which commercial cropping could be done to improvize the clove sector of the country.
\end{abstract}

Keywords: Syzygium, Genetic differentiation, RAPD, Clove oil, GC-MS.

\section{Introduction}

Clove [Sysygium aromaticum (Myrtaceae)], already well known as a spice, a food preservativeis becoming increasingly important for its medicinal values such as its anti-oxidant, anti-septic, local anesthetic, antiinflammatory, rubefacient (warming and soothing), carminative and anti-flatulent properties. They can be used to stimulate the mind as well as prevent nausea, diarrhoea, ease coughs, aid in digestion, and even treat conditions like malaria and cholera (Debjit Bhowmik et al., 2012). It represents one of the richest source of phenolic compounds such as eugenol, eugenol acetate and gallic acid. Eugenol is a phenyl-propanoids class of chemical compound which gives pleasant sweet aromatic fragrances to the clove bud. Eugenol has local anesthetic and anti-septic properties, hence useful in dental care essentials as well as in the treatment procedures (Shrivastava, 2006; Dhiman, 2006). In an earlier report, seven compounds have been isolated and characterized from Sysygium aromaticum namely oleanolic acid lactone, $\beta$-sitosterol, nigricin, flavaellagic acid, $2 \alpha$ hydroxyoleanolic acid, $3 \beta-$ hydroxy-11-oxo-olean-12-en-28-oic acid and $\beta$ sitosterol 3-O- $\beta$-D-glucopyranoside (Sabira Begum et al., 2014).

India is one of the producer, consumer, and exporter of clove, which is traded mainly in the form of whole-dried flower buds, as powder, as stem and in oil forms. Approximately, $72-90 \%$ of the essential oil extracted from cloves has Eugenol.

\section{*Corresponding Author:}

D. Saranya,

PhD Research Scholar,

PG and Research Department of Botany, Government Arts College,

Ooty, The Nilgiris, TamilNadu, India.

E-mail: pandeyamit0076@gmail.com
Other essential oil ingredients reported by (DebjitBowmik, et al., 2012) areacetyl eugenol, betacaryophyllene, vanillin, Crategolic acid, tannins, gallotannic acid, methyl salicylate (painkiller), Flavanoids, eugenin, kaempferol, rhamnetin, eugenitin, triterpenoids like oleanolic acid. The dried buds of cloves contain about 15-20 percent of essential oils, and the bulk of this is eugenol. A kilogram of dried buds provides about $150 \mathrm{ml}$. of eugenol. Recent reports on the medicinal value of cloves in treating a variety of ailments have further increased the demand for cloves all over the world. Consumer preference for organic products has also spurred the demand for clove at the same time, as with other organic products, especially those that are sold in oil form.

As part of our work, we used Random Amplification Polymorphic DNA (RAPD) to investigate the genetic diversity of $S$. aromaticum since morphological and morphometric study is time-consuming, laborious and consequently not suitable for large-scale application. DNA polymerase assay based on RAPD-PCR has been proved useful for analyzing the inter- and intraspecific genetic variations and phylogenetic relationships. RAPD technique is based on the amplification of a random DNA segment with a single primer of arbitrary nucleotide sequence and using the polymerase chain reaction. This technique 
is very rapid, simple and generates a reproducible fingerprint of the PCR products.

In addition, GC-MS technique is used to analyze the eugenol content of S.aromaticum. Gas Chromatography-Mass Spectrometry(GC-MS) is a hyphenated analytical technique that combines the separation properties of gas-liquid chromatography with the detection feature of mass spectrometry to identify different substances within a test sample. GC-MS has become a highly recommended tool for monitoring and tracking organic compounds in the test sample (Choi M H, Chung B C, 2014).

As the cultivation is concerned, in India clove is grown in the hilly areas of Tamilnadu, Kerala and Karnataka. The important clove growing districts in India are Nilgiris, Tirunrlveli, Kanyakumari, Nagercoil and Ramanathapuram districts of TamilNadu, Kozhikode, Kottayam, Kollam and Thiruvananthapuram districts of Kerala and South kanara district of Karnataka. Eventhough, Nilgiris is one of the producers of $S$. aromaticum, the genomic based research works to identify the genetic difference, high quality oil yielding varieties, etc. is very much limited which has to be concerned in favour of country's economic success. This could be possible by phylogenetic analysis.

The present research work describes the use ofRAPD to identify the genetic difference between the collected samples in comparison to the extracted oil quality using GC-MS technique to select high quality oil yielding variety for cropping.

\section{Materials and Methods}

Six clove leaf samples and flower buds from six different sampling points namely S1 (Athipali, Gudalur), S2 (Baaram, Gudalur), S3 (Maamaram, Kotagiri), S4 (Kallar, Coonoor), S5 (Barliar, Coonoor) and S6 (Muloor, Kotagiri) were studied for Genetic Diversity analysis using RAPD markers and oil quality analysis using GC-MS technique.

\section{DNA isolation protocol}

Total genomic DNA from the plant was isolated by N- Cetyl- N, N, N-trimethyl- ammonium bromide (CTAB) method. 2gm of the sample was weighed and transferred to a sterile mortar and pestle; $50 \mathrm{mg}$ of Polyvinylpyrolidone was added to the sample and ground well. $750 \mu$ l of extraction buffer was added and incubated at $65^{\circ} \mathrm{C}$ for 2 hours. Centrifuged at $10000 \mathrm{rpm}$ for $10 \mathrm{~min}$ at $4^{\circ} \mathrm{C}$. Clear solution was collected in a sterile microcentrifuge tube. Equal volumes of Chloroform: Isoamyl alcohol (24:1) was added. Centrifuged at $10000 \mathrm{rpm}$ for $10 \mathrm{~min}$. at $4{ }^{\circ} \mathrm{C}$. The aqueous phase was removed and taken in a sterile microcentrifuge tube. 0.6 volumes of isopropyl alcohol was added and incubated at room temperature for 1 hour. Centrifuged at $10000 \mathrm{rpm}$ for $10 \mathrm{~min}$. Pellet was washed in $500 \mu$ l of $70 \%$ ethanol. Centrifuged at $10000 \mathrm{rpm}$ for $10 \mathrm{~min}$ at room temperature. Pellet was dried and dissolved in $20 \mu$ l sterile distilled water.

\section{Lithium Chloride Precipitation:}

$10 \mu \mathrm{L}$ of the DNA sample was taken in a sterile $1.5 \mathrm{ml}$ tube and $90 \mu \mathrm{l}$ of sterile water was added and mixed well. Equal volume of Chloroform: isoamyl alcohol (24:1) was added to the tube and centrifuged at $10,000 \mathrm{rpm}$ for 10 minutes. The aqueous layer was transferred to a new $1.5 \mathrm{ml}$ tube and $200 \mu \mathrm{l}$ of $0.5 \mathrm{M}$ lithium chloride was added and incubated atC overnight. The tube was centrifuged at $10000 \mathrm{rpm}$ for $10 \mathrm{~min}$, the supernatant was discarded. To the pellet $500 \mu \mathrm{l}$ of $70 \%$ ethanol was added and centrifuged at $10000 \mathrm{rpm}$ for $10 \mathrm{~min}$. The pellet was dried and dissolved in $20 \mu \mathrm{l}$ sterile distilled water. The samples were stored at $-20 \mathrm{C}$.

\section{Quantification of Isolated DNA}

The quantity of the isolated DNA was checked in UV-VIS spectrophotometer (Vivaspec Biophotometer, Germany). From the stock $1 \mu \mathrm{l}$ DNA was mixed with $9-\mu l$ sterile distilled water to get 50 times dilution. The A260/A280 ratio was recorded to check the purity of DNA preparation.

\section{PCR Amplification}

PCR amplification was done in $20 \mu \mathrm{l}$ of reaction volume containing 1x PCR buffer (Kappa, SA); $\mathrm{MgCl}_{2}, 3 \mathrm{mM}$; dNTP mix, $0.25 \mathrm{mM}$; Taq DNA polymerase, $0.05 \mathrm{U}$; primer, $1 \mathrm{pmol}$ and template DNA, $50 \mathrm{ng}$. Sterile nuclease free water is used as negative control.

Agarose gel analysis of RAPD PCR (2\% Agarose)

2 gram of Agarose powder was measured and added to a $250 \mathrm{ml}$ conical flask. To the flask $100 \mathrm{ml}$ TAE (1X) buffer was added and mixed properly. The agarose was melted in a microwave or hot water bath until the solution became clear. The solution was allowed to cool to about $50-55^{\circ} \mathrm{C}$ with swirling of the flask occasionally to cool evenly. 1-2 $\mu$ l of Ethidium Bromide solution was added to the melted agarose gel and mixed well. Ends of the casting tray were sealed and combs were placed. Molten Agarose solution was poured into the casting tray without forming air bubbles and let to cool until it was solid. The combs were carefully pulled out and removed the tape and the gel was placed in the electrophoresis chamber. Sufficient amount of $1 \mathrm{X}$ TAE Buffer was added so that there is about $2-3 \mathrm{~mm}$ of buffer over the gel. $6 \mu \mathrm{l} 1$ of $6 \mathrm{x}$ Sample Loading Buffer was added to each $25 \mu \mathrm{l}$ of DNA sample. Each sample/ DNA ladder was carefully pipetted into separate wells in the gel. Electrode wires were connected to the power supply, making sure the positive (red) and negative (black) are correctly connected. Power supply was turned on to about 100 volts (should not exceed 5 volts / $\mathrm{cm}$ between electrodes). After sufficient run 
turn off the power pack, the gel was removed by using gloves. The DNA in the gel was visualized with U.V. light, photographed and documented using UV trans-illuminator.

\section{Clove Oil extraction and analysis}

Twenty gram of clove sample was powdered separately to extract oil by steam distillation. Clove oil consists of several compounds such as eugenol, which is the major component (85-90\%) and eugenol acetate (9-10\%). Eugenol has a boiling temperature of $254{ }^{\circ} \mathrm{C}$ and can be steam distilled from freshly ground clove. The powder was added to $150 \mathrm{~mL}$ of sterile distilled water in a $500 \mathrm{~mL}$ round bottom flask for distillation. The distillate was collected in a $125 \mathrm{~mL}$ E. flask. The distillate was extracted in ether. Continuous heating was provided from a heating mantle at a steady rate of $1 \mathrm{drop} / 2-3$ seconds. The water level was maintained and distilled until no oily layer is apparent. At least 200 $\mathrm{mL}$ of distillate was collected which is a waterclove oil mixture. To separate clove oil from water transferred distillate to a separation funnel and added $10 \mathrm{~mL}$ of saturated $\mathrm{NaCl}$ solution. Rinsed the condenser and receiving flask with $5 \mathrm{~mL}$ of ether and transferred this to the separation funnel. The mixture was allowed to separate after which the ether was separated out. The same was repeated twice to get more purified clove oil and it was stored in airtight glass vials. Thus, extracted oil was analysed for eugenol content by gas chromatography.

\section{Results and Discussions}

The confined reports on $S$. aromaticum stimulated to assess its molecular diversity using a PCR-based approach. The intrinsic genetic diversity in present study on $S$. aromaticum accessions was apparent from the analysis of their RAPD profiles and from dendrogram generates where all the accessions had unambiguously separated from each other. RAPD studies have been widely used for population genetic studies in both wild (Dikshit et al., 2007, Yao et al., 2008) and cultivated plants (Nagaoka and Ogihara, 1997; Sikdaret al., 2010). Many unique species-specific alleles were amplified by RAPD markers (Hardik K, et al., 2015). The six clove samples collected from different locations were analyzed for their genetic similarity using RAPD. Genomic DNA from six clove leaf samples was used to generate RAPD patterns with 10 RAPD markers which resulted in different banding pattern ranging from $100-3000 \mathrm{bp}$ shown in Figures $1-6$. To assess the degree of homology between shared bands, a different set of decamer primers was used on a minimal species set. The primers were OPAA01(5'AGACGGCTCC3'), OPAA02(5' GAGACCAGAC 3'), OPAA03(5' TTAGCGCCCC 3’), OPAA04(5' AGGACTGCTG 3'), OPAA05(5' GGCTTTAGCC 3'), OPAA06(5' GTGGGTGCCA 3’), OPAA07(5' CTACGCTCAC
3’), OPAA08(5' TCCGCAGTAG3'), OPAA09(5' AGATGGGCAG 3'), OPAA10(5' TGGTCGGGTG 3'). These OPAA primers were evaluated as part of a related experiment, and it is assumed that an estimate of homoplasy made with these primers will be applicable to RAPD generated patterns. For construction of a dendrogram with 167 bands scored for presence and absence as 1 and 0 respectively. Using PAST statistical software diversity between the samples was analyzed using paired group algorithm with Euclidean distance matrix.

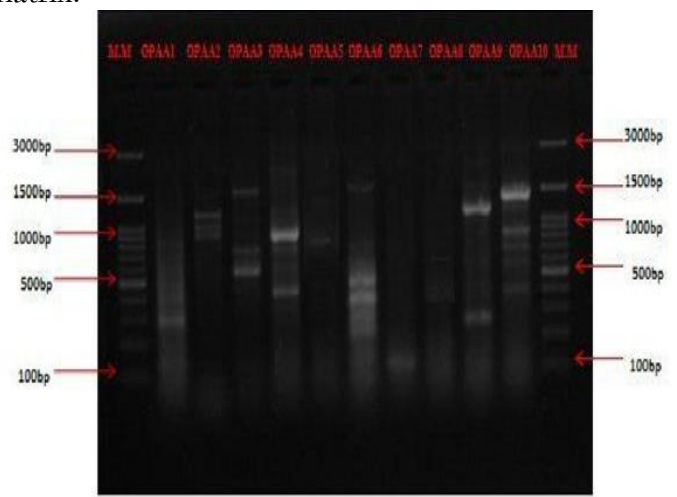

Figure 1.The banding patterns of sample 1 using 10 RAPD primers

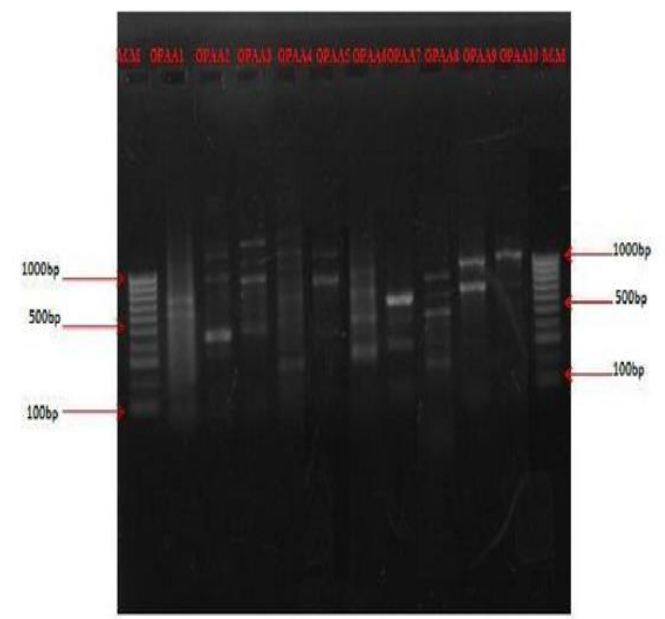

Figure 2. The banding patterns of sample 2 using 10 RAPD primers

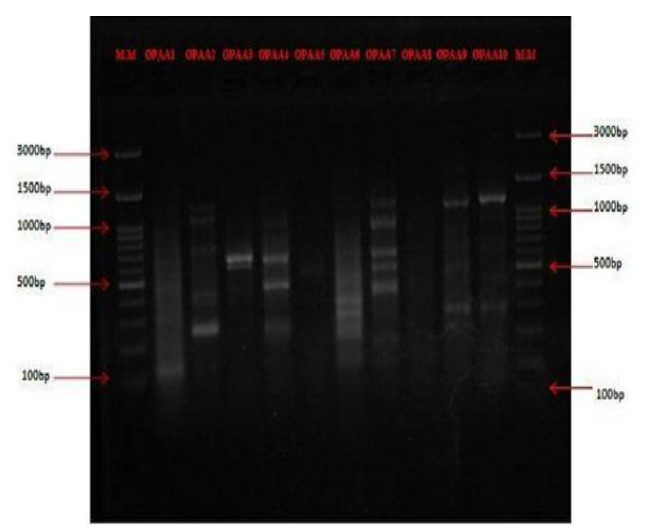

Figure 3.The banding patterns of sample 3 using 10 RAPD primers 


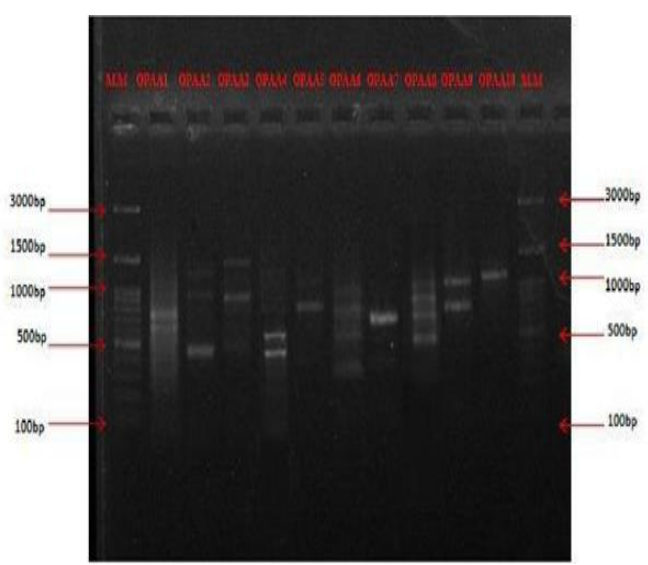

Figure 4. The banding patterns of sample 4 using 10 RAPD primers

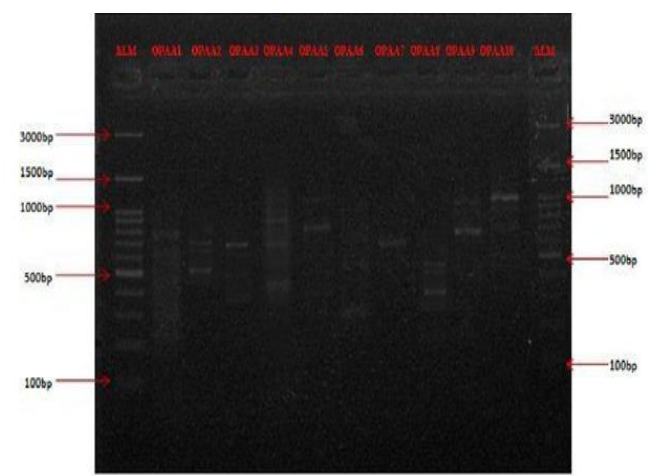

Figure 5. The banding patterns of sample 5 using 10 RAPD primers

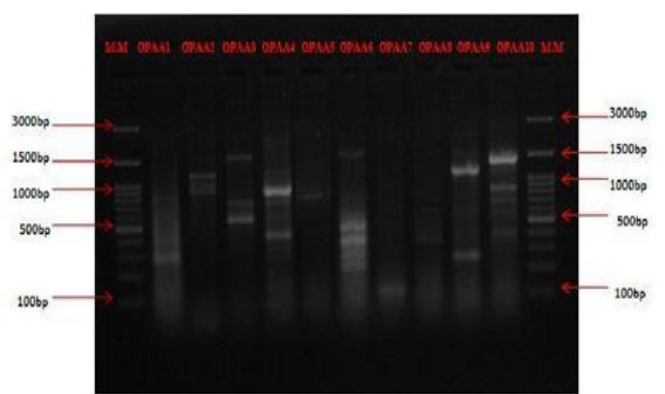

Figure 6. The banding patterns of sample 6 using 10 RAPD primers

The evolution of varieties in distinct agro-climatic zones demonstrates significant levels of variation in response to the selection pressure in the zones (Singh et al., 1998). It is therefore, not surprising to find significant levels of polymorphism among 6 genotypes of clove in RAPD. There are several reports confirming that RAPD technique has been applied to assess molecular polymorphism in Morchellasp. (Muhammad Irfanet al., 2017), pea (Simioniucet al., 2002). The success of our study in polymorphism is due to the use of number of randomly selected prescreened highly informative primers. Geographically isolated population accumulates genetic differences as they adapt to different environment. Genetic variation among clove genotypes based on RAPD analysis could be useful to select the best sample for cultivation. The dendrogram constructed by this method is shown in Figure 7. It revealed that the samples S1 and S6 are genetically more similar, whereas S2 is far distinct from all other samples. S3 shared a close relation with S1 and S6. Samples S4, S5 were separated out but are within the same node.

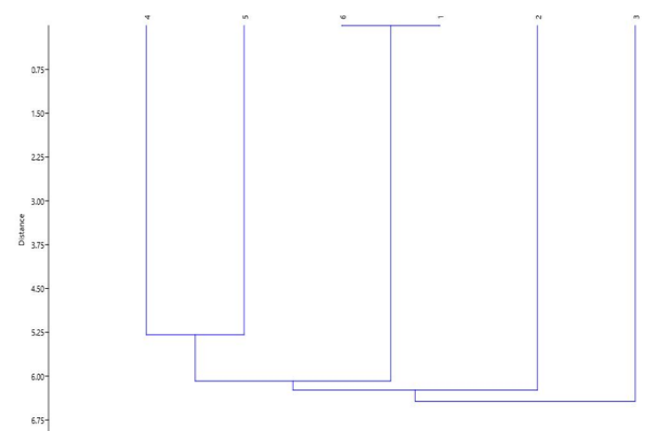

Figure 7: Dendrogram with Euclidean distance

When phytochemical studies of S.aromaticum is concerned, several group of compounds have been reported by different workers including $\beta$-sitosterol, nigricin, flavaellagic acid, $2 \alpha$ hydroxyoleanolic acid, $3 \beta$ hydroxyl-11-oxo-olean-12-en-28-oic acid, etc. (Sabira begum et al., 2014). Clove represents one of the major vegetal sources of phenolic compounds as flavanoids, hydroxybenzoic acids, hydroxycinamic acids and hydroxyphanylpropens. Eugenol, a phytogenic bioactive component is the most important compound in clove. Eugenol is the main bioactive compound of clove, which is found in concentrations ranging from 9381.70 to $14650.00 \mathrm{mg}$ per $100 \mathrm{~g}$ of fresh plant material (Neveu et al.,). Eugenol has been approved to encompass numerous beneficial aspects against a capacious spectrum of life threatening indispositions including oxidative stress, inflammation, hyperglycemia, elevated cholesterol level, neural disorders and cancer (Anees Ahmed Khalil, 2017). Therefore, in the present study clove oil was extracted and eugenol percentage was analysed to compare the genetic diversity with the oil quality. The eugenol percentage of the samples S1, S2, S3, S4, S5 and S6 are 42.96\%, 68.78\%, $55.95 \%, 71.17 \%, 74.04 \%$ and $73.47 \%$ respectively. The eugenol percentage analyzed from the extracted clove oil is all different for each sample though they are genetically similar.

Clove oil extraction was done using GC-MS technique. Gas chromatography mass spectrometry (GC-MS) has become firmly established as a key technological platform for secondary metabolite profiling in both plant and non-plant species (Kell DB, 2005). The main features of enhanced molecular ion, improved confidence in sample identification, significantly increased range of thermally labile and low volatility samples amenable for analysis, much faster analysis, improved sensitivity particularly for compounds that are hard to analyze are the compelling reasons to use the 
GC-MS in broad range of areas (ISO/IEC, 2005). GC-MS technique is a very useful for quality control, analytical research, impurity profiling and maintenance for human welfare and development (Chauhan A, 2014). Results of the present study revealed that samples S1 \& S6 are genetically more similar but their eugenol content was $42.96 \& 73.47$ $\%$ respectively. Similarly, S4, S5 has $71.17 \& 74.04$ $\%$ respectively and they are genetically similar. It is observed that they have small variation in the oil content. Whereas S2, S3 has 68.78 \& $55.95 \%$ eugenol content respectively.

\section{Conclusion}

From the collected data it is inferred that though the samples shared genetic similarity there observed a considerable difference in the eugenol content. This information needs to be correlated with the cultivation practices and the soil nutrition along with taxonomical and geographical information based on which commercial cropping could be done to improvise the clove sector of Nilgiris in welfare of the country to boost up the economy and development. Also, a specific primer could be designed in the obtained sequence as a SCAR marker for the high-quality oil yielding Nilgiris variety.

\section{References}

1. Anees Ahmed Khalil, Ubaid ur Rahman, Moazzam Rafiq Khan, Amna Sahar, Tariq Mehmood, Muneeb Khan. Essential oil eugenol: sources, extraction techniques and nutraceutical perspectives. RSC Advances 7 (2017): 32669.

2. Chauhan A, Goyal MK, Chauhan P. GC-MS technique and its analytical applications in science and technology. J Anal Bioanal Tech 5 (2014): 222.

3. Choi MH, Chung BC. Bringing GC-MS profiling of steroids into clinical applications. Mass Spectrom Rev. 34 (2014): 219-236.

4. Debjit Bhowmik, K P Sampath Kumar, Akhilesh Yadav, Shweta Srivastava, Shravan Paswan, Amit Sankar Dutta. Recent trends in Indian tranditional herb Sysygium aromaticum and its health benefits. Journal of pahrmacognosy and phytochemistry 1 (2012): 13-22.

5. Dikshit HK, Jhang T, Singh NK, Koundal KR, Bansal KC, Chandra N, Tickoo JL, Sharma TR. Genetic differentiation of Vigna species by RAPD, URP and SSR markers. Biol Plant 51.3 (2007): 451457.
6. Hardik K, Ranbir S, Sushil Kumar, Jigar G, Mukeshkumar. Detection of genetic variation in Ocimum species using RAPD and ISSR markers. Biotech 5 (2015):697-707.

7. ISO/IEC 17025. General requirements for competence of testing and calibration Laboratories. Paragraphs (2005) 5.5-5.6.

8. Kell DB, Brown M, Davey HM, Dunn WB, Spasic I, Oliver SG. Metabolic footprinting and systems biology: The medium is the message. Nat Rev Microbiol 3 (2005): 557-565.

9. Muhammad Irfan, Shuang Yang, Luo Yuxin, JiaXing Sun. Genetic diversity analysis of Morchella sp. By RAPD. Molecular Biology. (2017).

10. Neveu V, Perez-Jimenez J, Vos F et al., Phenol Explorer: an online comprehensive database on polyphenol contents in foods. doi: 10.1093/database/bap024[PMC free article].

11. Sabira Begum, Sara, Bina Shaheen Siddiqui, Rasheeda Khatoon, Faryal Aftab. Phytochemical studies on Syaygium aromaticum Linn. J. Chem.Soc. Pak 36 (2014): 512-517.

12. Sikdar B, Bhattacharya M, Mukherjee A, Banerjee A, Ghosh E, Ghosh B, Roy SC. Genetic diversity in important members of Cucurbitaceae using isozyme, RAPD and ISSR markers. Biol Plant 54 (2010):135140.

13. Singh AK, Smart J, Simpson CE, Raina SN. Genetic variation vis-à-vis molecular polymorphism in groundnut, Arachis hypogea L. Genet Resour Crop Evol 45 (1998):119-126.

14. Srivastava AK, Srivastava SK, Syamsundar KV. Volatile composition of Curcuma angustifolia Roxb. Rhizome from central and southern India. Flavour and Fragrance Journal 21 (2006): 423-426.

15. Yao H, Zhao Y, Chen DF, Chen JK, Zhao TS. ISSR primer screening and preliminary evaluation of genetic diversity in wild populations of Glycyrrhiza uralensis. Biol plant 52 (2008): 117-120.

\section{Cite this article as:}

Saranya D. and R. Ravi. Sysygium aromaticum: A subtropical tree scrutinized for the genetic differentiation among the altitudes of Nilgiris. Annals of Plant Sciences 7.4 (2018) pp. 2134-2138.

do $\mathrm{http} / /$ dx.doi.org/10.21746/aps.2018.7.4.10 